\title{
LAST EXIT BEFORE AN EXPONENTIAL TIME FOR SPECTRALLY NEGATIVE LÉVY PROCESSES
}

\author{
E. J. BAURDOUX, ${ }^{*}$ Universiteit Utrecht
}

\begin{abstract}
Chiu and Yin (2005) found the Laplace transform of the last time a spectrally negative Lévy process, which drifts to $\infty$, is below some level. The main motivation for the study of this random time stems from risk theory: what is the last time the risk process, modeled by a spectrally negative Lévy process drifting to $\infty$, is 0 ? In this paper we extend the result of Chiu and Yin, and we derive the Laplace transform of the last time, before an independent, exponentially distributed time, that a spectrally negative Lévy process (without any further conditions) exceeds (upwards or downwards) or hits a certain level. As an application, we extend a result found in Doney (1991).
\end{abstract}

Keywords: Spectrally negative Lévy process; exit problem; fluctuation theory; last passage time; risk theory

2000 Mathematics Subject Classification: Primary 60G99

Secondary 91B30

\section{Introduction}

The classical risk process, as introduced in [15], consists of a deterministic, positive drift $c$ plus a compound Poisson process which has only negative jumps. We denote by $\lambda>0$ the rate of the Poisson process and by $\mu$ the expected jump size. The main quantity of interest is the moment of ruin, i.e. the first time the risk process becomes negative. To ensure the moment of ruin is not almost surely finite, the net profit condition

$$
\frac{\lambda \mu}{c}<1
$$

is imposed. This condition ensures that the risk process drifts to $\infty$. Recently, various authors (see, for example, [4], [10], [11], and [12]) have replaced the classical risk process by a general spectrally negative Lévy process, which we shall denote by $X$. Also, in some cases, the moment of ruin may not be the most important quantity of the risk process. Indeed, consider the following scenario. Instead of going bankrupt when the risk process becomes negative, the firm has other funds which it can use to support the negative surplus for a while. For this reason, in [8], the Laplace transform was found for the last passage time at a certain level for the classical risk process. This was extended to the case of a general spectrally negative Lévy process in [5]. However, a more realistic quantity for study might be the last passage time below 0 before a fixed time $t$, i.e.

$$
S_{t}^{-}:=\sup \left\{0 \leq u \leq t: X_{u} \leq 0\right\} \quad \text { for } t \geq 0 .
$$

As is often the case, it turns out that it is easier to replace the fixed, deterministic time horizon by an independent, exponentially distributed random time. For $\theta \geq 0$, we denote by $\tilde{e}_{\theta}$ an

Received 13 April 2007; revision received 20 April 2009.

* Current address: Department of Statistics, London School of Economics, Houghton Street, London WC2A 2AE, UK. Email address: e.j.baurdoux@1se.ac.uk 
exponentially distributed random variable with parameter $\theta$. Here, we use the convention that an exponential random variable with parameter 0 is taken to be infinite with probability 1 . A Lévy process starting from $x \in \mathbb{R}$ (with respect to some probability space $\left(\Omega,\left\{\mathcal{F}_{t}\right\}_{t \geq 0}, \mathrm{P}_{x}\right)$ ) is said to be spectrally negative when it has no positive jumps and when it does not have monotone paths. We suppress the subscript in $\mathrm{P}_{x}$ when $x=0$. Now, define the random time

$$
\sigma_{\theta}^{-}=S_{\tilde{e}_{\theta}}^{-}=\sup \left\{0 \leq t \leq \tilde{e}_{\theta}: X_{t} \leq 0\right\},
$$

with the convention that $\sup \varnothing=0$. In the main result of this paper, Theorem 2 , we give the Laplace transform of $\sigma_{\theta}^{-}$. Using similar techniques, we also find the Laplace transform of

$$
\sigma_{\theta}^{+}=\sup \left\{0 \leq t \leq \tilde{e}_{\theta}: X_{t} \geq 0\right\}
$$

and of

$$
T_{\theta}=\sup \left\{0 \leq t \leq \tilde{e}_{\theta}: X_{t}=0\right\} .
$$

For convenience, we suppress the subscript when $\theta=0$. For spectrally negative Lévy processes drifting to $\infty$, the Laplace transform of $\sigma^{-}$was found in [5]. Trivially, in this case it holds that $T=\sigma^{-}$.

As an application of Theorem 2, we extend a result from [6]. In that paper it was proved that, for a spectrally negative stable process with index $\alpha$,

$$
\mathrm{P}\left(X_{t}=\bar{X}_{t}=t \text { for some } 0<t<\infty\right)=\frac{1}{\alpha},
$$

where $\bar{X}_{t}$ is the running supremum of $X$, i.e. $\bar{X}_{t}=\sup _{0 \leq s \leq t} X_{s}$. In the final section of this paper we find the Laplace transform of $\sup \left\{t \geq 0: X_{t}=\bar{X}_{t}=t\right\}$ for a general spectrally negative Lévy process.

Remark 1. The random times introduced above are not stopping times, as they depend on the future of the process $\left\{X_{t}\right\}_{t \geq 0}$.

\section{Preliminaries}

In this section we review some important properties of spectrally negative Lévy processes. For further details, we refer the reader to the books [2] and [13]. For a spectrally negative Lévy process $\left\{X_{t}\right\}_{t \geq 0}$, it holds that the Laplace exponent

$$
\psi(\lambda):=\log \mathrm{E}\left[\exp \left(\lambda X_{1}\right)\right], \quad \lambda \geq 0
$$

is well defined, convex, and infinitely differentiable on $(0, \infty)$. Furthermore, when $X$ is of bounded variation, we can express the Laplace exponent as

$$
\psi(\lambda)=d \lambda+\int_{(-\infty, 0)}\left(\mathrm{e}^{\lambda x}-1\right) \Pi(\mathrm{d} x),
$$

where $\Pi$ is the jump measure of $X$ and $d>0$ is called the drift.

For $q \geq 0$, the scale function $W^{(q)}(x)$ is defined as the continuous function on $[0, \infty)$ such that

$$
\int_{0}^{\infty} \mathrm{e}^{-\lambda x} W^{(q)}(x) \mathrm{d} x=\frac{1}{\psi(\lambda)-q} \quad \text { for any } \lambda>\Phi(q) .
$$


Here $\Phi$ denotes the right inverse of $\psi$. See, for example, Section VII.2 of [2] or Chapter 8 of [13] for a detailed study of the scale function. When $q=0$, we omit the superscript and write $W(x)$ instead. The function $W^{(q)}$ is extended to the negative half-line by setting $W^{(Q)}(x)=0$ when $x<0$. Note that $W^{(q)}$ is not necessarily continuous at 0 . In fact, it is not difficult to show that $W^{(q)}(0)=0$ when $X$ is of unbounded variation and $W^{(q)}(0)=1 / d$ when $X$ is of bounded variation with drift $d$. Furthermore, for $q \geq 0$, we define the function $Z^{(q)}$ by

$$
Z^{(q)}(x)=1+q \int_{0}^{x} W^{(q)}(y) \mathrm{d} y .
$$

Note that $Z^{(q)}(x)=1$ when $x \leq 0$. Integration by parts yields

$$
\int_{0}^{\infty} \mathrm{e}^{-\lambda x} Z^{(q)}(x) \mathrm{d} x=\frac{1}{\lambda}+\frac{q}{\lambda} \int_{0}^{\infty} \mathrm{e}^{-\lambda x} W^{(q)}(x) \mathrm{d} x=\frac{1}{\lambda}+\frac{q}{\lambda(\psi(\lambda)-q)} .
$$

For $a, b \in \mathbb{R}$, denote first passage times by

$$
\tau_{a}^{-}:=\inf \left\{t>0: X_{t} \leq a\right\}
$$

and

$$
\tau_{b}^{+}:=\inf \left\{t>0: X_{t} \geq b\right\}
$$

Also, we denote the first hitting time by

$$
T(a):=\inf \left\{t>0: X_{t}=a\right\} .
$$

Scale functions play a vital role in exit problems. For example, it holds that

$$
\mathrm{E}_{x}\left[\exp \left(-q \tau_{0}^{-}\right) \mathbf{1}_{\left\{\tau_{0}^{-}<\infty\right\}}\right]=Z^{(q)}(x)-W^{(q)}(x) \frac{q}{\Phi(q)},
$$

where, for the case in which $q=0$, the fraction $q / \Phi(q)$ is to be understood in the limiting sense. Expression (3) first appeared in the form of its Fourier transform in [7].

To derive our results concerning the last exit times, we also make use of potential measures. For spectrally negative Lévy processes, the $q$-potential measure $U^{(q)}(\mathrm{d} y)$, defined by

$$
\int_{0}^{\infty} \mathrm{e}^{-q t} \mathrm{P}\left(X_{t} \in \mathrm{d} y\right) \mathrm{d} t
$$

is absolutely continuous with respect to the Lebesgue measure, and a version of its density is given by

$$
u^{q}(y)=\Phi^{\prime}(q) \mathrm{e}^{-\Phi(q) y}-W^{(q)}(-y)
$$

see [3].

Since, for $c \geq 0$, the process $\left\{\exp \left(c X_{t}-\psi(c) t\right)\right\}$ is a martingale with mean 1 , we can introduce the change of measure

$$
\left.\frac{\mathrm{dP}^{c}}{\mathrm{dP}}\right|_{\mathcal{F}_{t}}=\exp \left(c X_{t}-\psi(c) t\right)
$$

The process $\left\{X_{t}\right\}_{t \geq 0}$ is still a spectrally negative Lévy process under $\mathrm{P}^{c}$, and we mark the Laplace exponent and scale functions of $X$ under $\mathrm{P}^{c}$ with the subscript $c$. It is straightforward to check that

$$
\psi_{c}(\lambda)=\psi(c+\lambda)-\psi(c) \text { for } \lambda \geq 0
$$


and by taking Laplace transforms we also find that

$$
W_{c}^{(q)}(x)=\mathrm{e}^{-c x} W^{(q+\psi(c))}(x) \quad \text { for } q \geq 0 .
$$

Furthermore, we readily check that, for $c, p \geq 0$,

$$
\begin{aligned}
\Phi_{c}(p) & =\sup \left\{x: \psi_{c}(x)=p\right\} \\
& =\sup \{x: \psi(x+c)=p+\psi(c)\} \\
& =\Phi(p+\psi(c))-c .
\end{aligned}
$$

For future reference, we also state the following result, the proof of which is given in Appendix A.

Lemma 1. For $q>0$ and $\lambda \geq-\Phi(q)$,

$$
\int_{[0, \infty)} \mathrm{e}^{-\lambda x} W_{\Phi(q)}(\mathrm{d} x)=\frac{\lambda}{\psi(\Phi(q)+\lambda)-q},
$$

where the right-hand side is to be interpreted in the limiting sense as $\Phi^{\prime}(q)$ for the case in which $\lambda=0$.

Finally, we collect a couple of well-known expressions for first exit problems which we shall use throughout this paper.

Lemma 2. For $x>0$ and $u, v \geq 0$,

$$
\mathrm{E}_{x}\left[\exp \left(-u \tau_{0}^{-}+v X_{\tau_{0}^{-}}\right) \mathbf{1}_{\left\{\tau_{0}^{-}<\infty\right\}}\right]=\mathrm{e}^{v x}\left(Z_{v}^{(p)}(x)-W_{v}^{(p)}(x) \frac{p}{\Phi_{v}(p)}\right),
$$

where $p=u-\psi(v)$. For the case in which $u=\psi(v)$, the fraction $p / \Phi_{v}(p)$ is to be interpreted in the limiting sense as

$$
\lim _{u \rightarrow \psi(v)} \frac{u-\psi(v)}{\Phi_{v}(u-\psi(v))}=\frac{1}{\Phi^{\prime}(\psi(v))}
$$

For $x<0$ and $q \geq 0$,

$$
\mathrm{E}_{x}\left[\exp \left(-q \tau_{0}^{+}\right) \mathbf{1}_{\left\{\tau_{0}^{+}<\infty\right\}}\right]=\mathrm{e}^{\Phi(q) x} .
$$

Finally, for $x>0$ and $q \geq 0$,

$$
\mathrm{E}_{x}\left[\mathrm{e}^{-q T(0)} \mathbf{1}_{\{T(0)<\infty\}}\right]=\mathrm{e}^{\Phi(q) x}-\psi^{\prime}(\Phi(q)) W^{(q)}(x),
$$

and the case in which $x=0$ is given by $1-\left(d \Phi^{\prime}(0)\right)^{-1}$ when $X$ has bounded variation with drift $d$.

Expression (7) follows after a change of measure from (3). Using the fact that the process $\left\{\exp \left(-q t+\Phi(q) X_{t}\right)\right\}_{t \geq 0}$ is a martingale, we can deduce (9). Finally, (10) was established in the form of its Laplace transform in Theorem 1 of [6].

\section{Main result}

Not surprisingly, scale functions also play a predominant role when considering last exit times. 
Theorem 1. ([5, Theorem 3.1].) Suppose that $\psi^{\prime}(0)>0$. Then, for $q>0$ and $x \in \mathbb{R}$,

$$
\mathrm{E}_{x}\left[\mathrm{e}^{-q \sigma^{-}} \mathbf{1}_{\left\{\sigma^{-}>0\right\}}\right]=\Phi^{\prime}(q) \psi^{\prime}(0) \mathrm{e}^{\Phi(q) x}-\psi^{\prime}(0) W^{(q)}(x) .
$$

In this paper we extend this result by considering last passage below a certain level before an independent, exponentially distributed time (as well as last passage above and last hit of a fixed level). We state the main result of this paper.

Theorem 2. For $q, \theta \geq 0$ and $x \in \mathbb{R}$,

$$
\begin{aligned}
\mathrm{E}_{x}\left[\exp \left(-q \sigma_{\theta}^{-}\right)\right]= & 1+\mathrm{e}^{\Phi(q+\theta) x} \Phi^{\prime}(q+\theta)\left(\frac{\theta}{\Phi(\theta)}-\frac{\theta}{\Phi(q+\theta)}\right)+\frac{\theta}{q+\theta} Z^{(q+\theta)}(x) \\
& -Z^{(\theta)}(x)+\frac{\theta}{\Phi(\theta)}\left(W^{(\theta)}(x)-W^{(q+\theta)}(x)\right)
\end{aligned}
$$

Furthermore,

$$
\begin{aligned}
\mathrm{E}_{x}\left[\exp \left(-q \sigma_{\theta}^{+}\right)\right]= & \frac{q}{q+\theta} Z^{(q+\theta)}(x)-\mathrm{e}^{\Phi(\theta) x} Z_{\Phi(\theta)}^{(q)}(x)+\frac{\theta}{\theta+q} \\
& +\mathrm{e}^{\Phi(q+\theta) x} \frac{q \Phi(\theta) \Phi^{\prime}(q+\theta)}{\Phi(q+\theta)(\Phi(q+\theta)-\Phi(\theta))} .
\end{aligned}
$$

Finally,

$$
\mathrm{E}_{x}\left[\exp \left(-q T_{\theta}\right)\right]=1-\mathrm{e}^{\Phi(\theta) x}+\frac{1}{\Phi^{\prime}(\theta)}\left(W^{(\theta)}(x)-W^{(q+\theta)}(x)\right)+\frac{\Phi^{\prime}(q+\theta)}{\Phi^{\prime}(\theta)} \mathrm{e}^{\Phi(q+\theta) x} .
$$

Combined with the strong Markov property, Theorem 2 allows us to readily obtain expressions for the joint Laplace transform of the first and last exit times.

Corollary 1. Let $p, q \geq 0$. When $X$ does not oscillate,

$$
\mathrm{E}_{x}\left[\mathrm{e}^{-p T(0)-q T} \mathbf{1}_{\{T(0)<\infty\}}\right]=\frac{\Phi^{\prime}(q)}{\Phi^{\prime}(0)}\left(\mathrm{e}^{\Phi(p+q) x}-\frac{1}{\Phi^{\prime}(p+q)} W^{(p+q)}(x)\right) .
$$

When $X$ drifts to $-\infty$ and $x<0$,

$$
\mathrm{E}_{x}\left[\exp \left(-p \tau_{0}^{+}-q \sigma^{+}\right) \mathbf{1}_{\left\{\tau_{0}^{+}<\infty\right\}}\right]=\frac{q \Phi(0) \Phi^{\prime}(q)}{\Phi(q)(\Phi(q)-\Phi(0))} \mathrm{e}^{\Phi(p+q) x} .
$$

When $X$ drifts to $+\infty$,

$\mathrm{E}_{x}\left[\exp \left(-p \tau_{0}^{-}-q \sigma^{-}\right) \mathbf{1}_{\left\{\tau_{0}^{-}<\infty\right\}}\right]=\frac{\Phi^{\prime}(q)}{\Phi^{\prime}(0)}\left(\mathrm{e}^{\Phi(q) x} Z_{\Phi(q)}^{(p)}(x)-\frac{p}{\Phi(p+q)-\Phi(q)} W^{(p+q)}(x)\right)$.

Proof. The third equality has already been obtained in [5]. We only prove (15), as the proofs of the other claims are similar. Suppose that $X$ drifts to $\infty$. Then $\Phi(0)=0$ and, from the strong Markov property applied at $T(0)$ and (10), we find that

$$
\begin{aligned}
\mathrm{E}_{x}\left[\mathrm{e}^{-p T(0)-q T} \mathbf{1}_{\{T(0)<\infty\}}\right] & =\mathrm{E}_{x}\left[\mathrm{e}^{-(p+q) T(0)} \mathbf{1}_{\{T(0)<\infty\}} \mathrm{E}\left[\mathrm{e}^{-q T}\right]\right] \\
& =\frac{\Phi^{\prime}(q)}{\Phi^{\prime}(0)}\left(\mathrm{e}^{\Phi(p+q) x}-\frac{1}{\Phi^{\prime}(p+q)} W^{(p+q)}(x)\right) .
\end{aligned}
$$


Remark 2. Note that Theorem 1 follows by taking $p=0$ in (16) (or in (15)).

When $X$ is a stable process, we can invert the double Laplace transform in (14) (when $x=0$ ) and retrieve the known result that, for each $t \geq 0$, the random variable defined, analogously to (1), by

$$
S_{t}:=\sup \left\{0 \leq u \leq t: X_{u}=0\right\}, \quad t \geq 0,
$$

is distributed according to the so-called generalized arcsine law. When $\alpha=2$, this is the wellknown arcsine law for Brownian motion (see, for example, [14, Chapter VI]). In fact, using the scaling property of stable processes, the following result can be shown to hold for any stable process with index $\alpha>1$ (i.e. not only in the spectrally negative case). We refer the reader to Theorem VIII.12 of [2] for the proof in the general case.

Corollary 2. Suppose that $X$ is a spectrally negative stable process with index $\alpha \in(1,2]$. Then, for $0 \leq s \leq t$,

$$
\mathrm{P}\left(S_{t} \in \mathrm{d} s\right)=\frac{\sin (\pi / \alpha)}{\pi} s^{-1 / \alpha}(t-s)^{-1+1 / \alpha} \mathrm{d} s .
$$

Also, the distribution of $S_{t}^{-}$is given by

$$
\mathrm{P}\left(S_{t}^{-} \in \mathrm{d} s\right)=\frac{1}{\alpha} \frac{\sin (\pi / \alpha)}{\pi} s^{-1 / \alpha}(t-s)^{-1+1 / \alpha} \mathrm{d} s+\left(1-\frac{1}{\alpha}\right) \delta_{t}(\mathrm{~d} s),
$$

where $\delta_{t}$ is the Dirac measure at $t$.

Proof. When $X$ is a spectrally negative stable process of index $\alpha$, it holds that (without loss of generality) $\psi(\lambda)=\lambda^{\alpha}$ for $\alpha \geq 0$ and, thus, $\Phi(q)=q^{1 / \alpha}$ for $q \geq 0$. It is straightforward to check that

$$
\int_{0}^{\infty} \int_{s}^{\infty} \mathrm{e}^{-q s-\theta t} s^{-1 / \alpha}(t-s)^{-1+1 / \alpha} \mathrm{d} t \mathrm{~d} s=\Gamma\left(\frac{1}{\alpha}\right) \Gamma\left(1-\frac{1}{\alpha}\right) \theta^{-1 / \alpha}(\theta+q)^{-1+1 / \alpha} .
$$

From (14) we now deduce (17), and (18) follows in a similar way from (11).

\section{Proof of Theorem 2}

For $q \geq 0$, we denote by $e_{q}$ an exponentially distributed random variable with parameter $q$ which is independent of $X$ and $\tilde{e}_{\theta}$. We split the proof of Theorem 2 into different parts.

Proof of (11). Let

$$
A^{+}=\left\{\tilde{e}_{\theta} \geq e_{q}, X_{e_{q}}>0, X_{s}>0 \text { for all } s \in\left[e_{q}, \tilde{e}_{\theta}\right]\right\} .
$$

We can then write the event $\left\{\sigma_{\theta}^{-}<e_{q}\right\}$ as a disjoint union

$$
\left\{\sigma_{\theta}^{-}<e_{q}\right\}=\left\{\tilde{e}_{\theta}<e_{q}\right\} \cup A^{+} .
$$

We thus have

$$
\mathrm{E}_{x}\left[\exp \left(-q \sigma_{\theta}^{-}\right)\right]=\mathrm{P}_{x}\left(\sigma_{\theta}^{-}<e_{q}\right)=\mathrm{P}\left(\tilde{e}_{\theta}<e_{q}\right)+\mathrm{P}_{x}\left(A^{+}\right)=\frac{\theta}{\theta+q}+\mathrm{P}_{x}\left(A^{+}\right) .
$$


Now, for $x \leq 0$,

$$
\begin{aligned}
\mathrm{P}_{x}\left(A^{+}\right) & =q \mathrm{E}_{x}\left[\int_{0}^{\infty} \mathrm{e}^{-q t} \mathbf{1}_{\left\{\tilde{e}_{\theta} \geq t\right\}} \mathbf{1}_{\left\{X_{s}>0 \text { for all } s \in\left[t, \tilde{e}_{\theta}\right]\right\}} \mathrm{d} t\right] \\
& =q \int_{0}^{\infty} \mathrm{e}^{-(q+\theta) t} \int_{(0, \infty)} \mathrm{P}_{x}\left(X_{t} \in \mathrm{d} y\right) \mathrm{P}_{y}\left(\tau_{0}^{-}>\tilde{e}_{\theta}\right) \mathrm{d} t \\
& =q \int_{(0, \infty)} u^{(q+\theta)}(y-x)\left(1-\mathrm{E}_{y}\left[\exp \left(-\theta \tau_{0}^{-}\right)\right]\right) \mathrm{d} y \\
& =q \int_{0}^{\infty} \Phi^{\prime}(q+\theta) \mathrm{e}^{-\Phi(q+\theta)(y-x)}\left(1-Z^{(\theta)}(y)+W^{(\theta)}(y) \frac{\theta}{\Phi(\theta)}\right) \mathrm{d} y \\
& =q \Phi^{\prime}(q+\theta) \mathrm{e}^{\Phi(q+\theta) x}\left(\frac{1}{\Phi(q+\theta)}-\frac{1}{\Phi(q+\theta)}-\frac{\theta}{q \Phi(q+\theta)}+\frac{\theta}{\Phi(\theta)} \frac{1}{q}\right) \\
& =\Phi^{\prime}(q+\theta) \mathrm{e}^{\Phi(q+\theta) x}\left(\frac{\theta}{\Phi(\theta)}-\frac{\theta}{\Phi(q+\theta)}\right),
\end{aligned}
$$

where the second equality follows from the Markov property and the lack of memory property of the exponential distribution, the fourth equality follows from (4) and (7), and the fifth equality follows from (2) and the definition of $W^{(q)}$. Hence,

$$
\mathrm{E}_{x}\left[\exp \left(-q \sigma_{\theta}^{-}\right)\right]=\frac{\theta}{\theta+q}+\Phi^{\prime}(q+\theta) \mathrm{e}^{\Phi(q+\theta) x}\left(\frac{\theta}{\Phi(\theta)}-\frac{\theta}{\Phi(q+\theta)}\right) \text { for } x \leq 0 .
$$

Next, let $x>0$. In this case, $\sigma_{\theta}^{-}$is equal to 0 whenever $X$ does not become negative before $\tilde{e}_{\theta}$. Taking this into account, we refine (19) and write the event $\left\{\sigma_{\theta}^{-}<e_{q}\right\}$ as a disjoint union:

$$
\begin{aligned}
\left\{\sigma_{\theta}^{-}<e_{q}\right\} & =\left\{\tilde{e}_{\theta}<e_{q}\right\} \cup\left\{\sigma_{\theta}^{-}=0, \tilde{e}_{\theta} \geq e_{q}\right\} \cup\left\{\sigma_{\theta}^{-} \in\left(0, e_{q}\right), \tilde{e}_{\theta} \geq e_{q}\right\} \\
& =\left\{\tilde{e}_{\theta}<e_{q}\right\} \cup\left\{\tau_{0}^{-}>\tilde{e}_{\theta}, \tilde{e}_{\theta} \geq e_{q}\right\} \cup\left(\left\{\tau_{0}^{-}<\tilde{e}_{\theta}\right\} \cap A^{+}\right) .
\end{aligned}
$$

We thus have

$$
\mathrm{E}_{x}\left[\exp \left(-q \sigma_{\theta}^{-}\right)\right]=\frac{\theta}{\theta+q}+\mathrm{P}_{x}\left(\tau_{0}^{-}>\tilde{e}_{\theta} \geq e_{q}\right)+\mathrm{P}_{x}\left(\tau_{0}^{-}<\tilde{e}_{\theta}, A^{+}\right),
$$

and deduce that

$$
\begin{aligned}
\mathrm{P}_{x}\left(\tau_{0}^{-}>\tilde{e}_{\theta}, \tilde{e}_{\theta} \geq e_{q}\right)= & \mathrm{E}_{x}\left[\int_{0}^{\infty} \theta \mathrm{e}^{-\theta y} \mathbf{1}_{\left\{\tau_{0}^{-}>y>e_{q}\right\}} \mathrm{d} y\right] \\
= & \mathrm{E}_{x}\left[\left(\exp \left(-\theta e_{q}\right)-\exp \left(-\theta \tau_{0}^{-}\right)\right) \mathbf{1}_{\left\{\tau_{0}^{-}>e_{q}\right\}}\right] \\
= & \mathrm{E}_{x}\left[\int_{0}^{\infty}\left(\mathrm{e}^{-\theta z}-\exp \left(-\theta \tau_{0}^{-}\right)\right) q \mathrm{e}^{-q z} \mathbf{1}_{\left\{\tau_{0}^{-}>z\right\}} \mathrm{d} z\right] \\
= & \frac{q}{q+\theta}+\frac{\theta}{q+\theta} \mathrm{E}_{x}\left[\exp \left(-(q+\theta) \tau_{0}^{-}\right)\right]-\mathrm{E}_{x}\left[\exp \left(-\theta \tau_{0}^{-}\right)\right] \\
= & \frac{q}{q+\theta}+\frac{\theta}{q+\theta}\left(Z^{(q+\theta)}(x)-\frac{q+\theta}{\Phi(q+\theta)} W^{(q+\theta)}(x)\right) \\
& -Z^{(\theta)}(x)+\frac{\theta}{\Phi(\theta)} W^{(\theta)}(x),
\end{aligned}
$$


where (3) was used for the last equality. For $\theta, q \geq 0$, define

$$
\lambda(\theta, q):=\Phi^{\prime}(q+\theta)\left(\frac{\theta}{\Phi(\theta)}-\frac{\theta}{\Phi(q+\theta)}\right) .
$$

From the strong Markov property applied at $\tau_{0}^{-}$, the memoryless property of the exponential distribution, (7), and (20), we deduce that

$$
\begin{aligned}
\mathrm{P}_{x}\left(\tau_{0}^{-}<\tilde{e}_{\theta}, A^{+}\right) & =\mathrm{E}_{x}\left[\mathbf{1}_{\left\{\tau_{0}^{-}<e_{q} \wedge \tilde{e}_{\theta}\right\}} \mathrm{P}_{X_{\tau^{-}}}\left(A^{+}\right)\right] \\
& =\lambda(\theta, q) \mathrm{E}_{x}\left[\exp \left(\Phi(q+\theta) X_{\tau_{0}^{-}}\right) \mathbf{1}_{\left\{\tau_{0}^{-}<e_{q} \wedge \tilde{e}_{\theta}\right\}}\right] \\
& =\lambda(\theta, q) \mathrm{E}_{x}\left[\exp \left(-(q+\theta) \tau_{0}^{-}+\Phi(q+\theta) X_{\tau_{0}^{-}}\right) \mathbf{1}_{\left\{\tau_{0}^{-}<\infty\right\}}\right] \\
& =\lambda(\theta, q) \mathrm{e}^{\Phi(q+\theta) x}\left(1-W_{\Phi(q+\theta)}(x) \frac{1}{\Phi^{\prime}(q+\theta)}\right),
\end{aligned}
$$

where we also used the fact that $e_{q} \wedge \tilde{e}_{\theta}$ is exponentially distributed with parameter $q+\theta$ for the third equality. From (5) we know that $\mathrm{e}^{\Phi(p+q) x} W_{\Phi(p+q)}(x)=W^{(p+q)}(x)$ and, thus, (11) follows.

Proof of (13). We can write the event $\left\{\sigma_{\theta}^{+}<e_{q}\right\}$ as a disjoint union:

$$
\left\{\tilde{e}_{\theta}<e_{q}\right\} \cup A^{-},
$$

where

$$
A^{-}=\left\{\tilde{e}_{\theta}>e_{q}, X_{s}<0 \text { for all } s \in\left[e_{q}, \tilde{e}_{\theta}\right]\right\}
$$

We thus have

$$
\mathrm{E}_{x}\left[\exp \left(-q \sigma_{\theta}^{+}\right)\right]=\mathrm{P}_{x}\left(\sigma_{\theta}^{+}<e_{q}\right)=\frac{\theta}{\theta+q}+\mathrm{P}_{x}\left(A^{-}\right) .
$$

Let $x \geq 0$. Then

$$
\begin{aligned}
\mathrm{P}_{x}\left(A^{-}\right) & =q \mathrm{E}_{x}\left[\int_{0}^{\infty} \mathrm{e}^{-q t} \mathbf{1}_{\left\{\tilde{e}_{\theta}>t\right\}} \mathbf{1}_{\left\{X_{s}<0 \text { for all } s \in\left[t, \tilde{e}_{\theta}\right]\right\}} \mathrm{d} t\right] \\
& =q \int_{0}^{\infty} \mathrm{e}^{-(q+\theta) t} \int_{(-\infty, 0)} \mathrm{P}_{x}\left(X_{t} \in \mathrm{d} y\right) \mathrm{P}_{y}\left(\tau_{0}^{+}>\tilde{e}_{\theta}\right) \mathrm{d} t \\
& =q \int_{(-\infty, 0)} u^{(q+\theta)}(y-x) \mathrm{P}_{y}\left(\tau_{0}^{+}>\tilde{e}_{\theta}\right) \mathrm{d} y \\
& =q \int_{(-\infty, 0)}\left(\Phi^{\prime}(q+\theta) \mathrm{e}^{\Phi(q+\theta)(x-y)}-W^{(q+\theta)}(x-y)\right)\left(1-\mathrm{e}^{\Phi(\theta) y}\right) \mathrm{d} y \\
& =q \int_{(0, \infty)}\left(\Phi^{\prime}(q+\theta)-W_{\Phi(q+\theta)}(x+y)\right) \mathrm{e}^{\Phi(q+\theta)(x+y)}\left(1-\mathrm{e}^{-\Phi(\theta) y}\right) \mathrm{d} y \\
& =q \mathrm{e}^{\Phi(q+\theta) x} \int_{(0, \infty)} \int_{x+y}^{\infty} W_{\Phi(q+\theta)}^{\prime}(z)\left(\mathrm{e}^{\Phi(q+\theta) y}-\mathrm{e}^{(\Phi(q+\theta)-\Phi(\theta)) y}\right) \mathrm{d} z \mathrm{~d} y \\
& =q \mathrm{e}^{\Phi(q+\theta) x} \int_{(x, \infty)}^{z-x} \int_{0}^{z} W_{\Phi(q+\theta)}^{\prime}(z)\left(\mathrm{e}^{\Phi(q+\theta) y}-\mathrm{e}^{(\Phi(q+\theta)-\Phi(\theta)) y}\right) \mathrm{d} y \mathrm{~d} z
\end{aligned}
$$

where the second equality follows from the Markov property and the lack of memory property of the exponential distribution, the fourth equality follows from (4) and (9), the fifth equality 
follows from (5), the penultimate equality follows from $W_{\Phi(q+\theta)}(\infty)=\Phi^{\prime}(q+\theta)$ (see (33) in Appendix A), and the last equality follows from an application of Fubini's theorem. Define

$$
f(x, z):=\frac{\mathrm{e}^{\Phi(q+\theta)(z-x)}}{\Phi(q+\theta)}-\frac{\mathrm{e}^{(\Phi(q+\theta)-\Phi(\theta))(z-x)}}{\Phi(q+\theta)-\Phi(\theta)}+\frac{\Phi(\theta)}{\Phi(q+\theta)(\Phi(q+\theta)-\Phi(\theta))} .
$$

Integration by parts yields

$$
\begin{aligned}
\int_{(x, \infty)} & \int_{0}^{z-x} W_{\Phi(q+\theta)}^{\prime}(z)\left(\mathrm{e}^{\Phi(q+\theta) y}-\mathrm{e}^{(\Phi(q+\theta)-\Phi(\theta)) y}\right) \mathrm{d} y \mathrm{~d} z \\
= & \int_{[0, \infty)} f(x, z) W_{\Phi(q+\theta)}(\mathrm{d} z)-\int_{[0, x]} f(x, z) W_{\Phi(q+\theta)}(\mathrm{d} z) \\
= & \int_{[0, \infty)} f(x, z) W_{\Phi(q+\theta)}(\mathrm{d} z) \\
& +\int_{0}^{x} W_{\Phi(q+\theta)}(z)\left(\mathrm{e}^{\Phi(q+\theta)(z-x)}-\mathrm{e}^{(\Phi(q+\theta)-\Phi(\theta))(z-x)}\right) \mathrm{d} z \\
= & \int_{[0, \infty)} f(x, z) W_{\Phi(q+\theta)}(\mathrm{d} z)+\mathrm{e}^{-\Phi(q+\theta) x} \frac{Z^{(q+\theta)}(x)-1}{q+\theta} \\
& -\mathrm{e}^{(\Phi(\theta)-\Phi(q+\theta)) x} \frac{Z_{\Phi(\theta)}^{(q)}(x)-1}{q} .
\end{aligned}
$$

Using this expression and invoking (21) and (22), we conclude that

$$
\begin{aligned}
\mathrm{E}_{x}\left[\exp \left(-q \sigma_{\theta}^{+}\right)\right]= & \frac{\theta}{q+\theta}+\mathrm{P}_{x}\left(A^{-}\right) \\
= & \frac{\theta}{q+\theta}+q \mathrm{e}^{\Phi(q+\theta) x} \int_{[0, \infty)} f(x, z) W_{\Phi(q+\theta)}(\mathrm{d} z) \\
& +\frac{q}{q+\theta}\left(Z^{(q+\theta)}(x)-1\right)-\mathrm{e}^{\Phi(\theta) x}\left(Z_{\Phi(\theta)}^{(q)}(x)-1\right) \\
= & \frac{\theta}{q+\theta}+\frac{q}{q+\theta}-\frac{q}{\Phi(q+\theta)-\Phi(\theta)} \frac{\Phi(\theta)-\Phi(q+\theta)}{\psi(\Phi(\theta))-(q+\theta)} \mathrm{e}^{\Phi(\theta) x} \\
& +\frac{q \Phi(\theta)}{\Phi(q+\theta)(\Phi(q+\theta)-\Phi(\theta))} W_{\Phi(q+\theta)}(\infty) \mathrm{e}^{\Phi(q+\theta) x} \\
& +\frac{q}{q+\theta}\left(Z^{(q+\theta)}(x)-1\right)-\mathrm{e}^{\Phi(\theta) x}\left(Z_{\Phi(\theta)}^{(q)}(x)-1\right) \\
= & \frac{q}{q+\theta} Z^{(q+\theta)}(x)-\mathrm{e}^{\Phi(\theta) x} Z_{\Phi(\theta)}^{(q)}(x)+\frac{\theta}{\theta+q} \\
& +\mathrm{e}^{\Phi(q+\theta) x} \frac{q \Phi(\theta) \Phi^{\prime}(q+\theta)}{\Phi(q+\theta)(\Phi(q+\theta)-\Phi(\theta))},
\end{aligned}
$$

where the third equality follows from an application of Lemma 1 and the last equality is again a consequence of $W_{\Phi(q+\theta)}(\infty)=\Phi^{\prime}(q+\theta)$. Note that (23) implies that

$$
\mathrm{P}\left(A^{-}\right)=\frac{q \Phi(\theta) \Phi^{\prime}(q+\theta)}{\Phi(q+\theta)(\Phi(q+\theta)-\Phi(\theta))}-\frac{\theta}{\theta+q} .
$$


Next, let $x<0$. We decompose $\left\{e_{q}>\sigma_{\theta}^{+}\right\}$as

$$
\left\{\sigma_{\theta}^{+}<e_{q}\right\}=\left\{\tilde{e}_{\theta}<e_{q}\right\} \cup\left\{\tau_{0}^{+}>\tilde{e}_{\theta}, \tilde{e}_{\theta} \geq e_{q}\right\} \cup\left(\left\{\tau_{0}^{+}<\tilde{e}_{\theta}\right\} \cap A^{-}\right) .
$$

As before, we deduce from the strong Markov property, the memoryless property of the exponential distribution, (9), and (24) that

$$
\begin{aligned}
& \mathrm{E}_{x}\left[\exp \left(-q \sigma_{\theta}^{+}\right)\right]=\frac{\theta}{\theta+q}+\mathrm{P}_{x}\left(\tau_{0}^{+}>\tilde{e}_{\theta}>e_{q}\right)+\mathrm{P}_{x}\left(\sigma_{\theta}^{+} \in\left(0, e_{q}\right), \tilde{e}_{\theta} \geq e_{q}\right) \\
& =\frac{\theta}{\theta+q}+\mathrm{E}_{x}\left[\int_{0}^{\infty} \theta \mathrm{e}^{-\theta y} \mathbf{1}_{\left\{\tau_{0}^{+}>y>e_{q}\right\}} \mathrm{d} y\right]+\mathrm{E}_{x}\left[\mathbf{1}_{\left\{\tau_{0}^{+}<e_{q} \wedge \tilde{e}_{\theta}\right\}} \mathrm{P}\left(A^{-}\right)\right] \\
& =\frac{\theta}{\theta+q}+\mathrm{E}_{x}\left[\left(\exp \left(-\theta e_{q}\right)-\exp \left(-\theta \tau_{0}^{+}\right)\right) \mathbf{1}_{\left\{\tau_{0}^{-}>e_{q}\right\}}\right] \\
& +\left(\frac{q \Phi(\theta) \Phi^{\prime}(q+\theta)}{\Phi(q+\theta)(\Phi(q+\theta)-\Phi(\theta))}-\frac{\theta}{\theta+q}\right) \mathrm{P}_{x}\left(\tau_{0}^{+}<e_{q} \wedge \tilde{e}_{\theta}\right) \\
& =\frac{\theta}{\theta+q}+\mathrm{E}_{x}\left[\int_{0}^{\infty} q \mathrm{e}^{-q t}\left(\mathrm{e}^{-\theta t}-\exp \left(-\theta \tau_{0}^{+}\right)\right) \mathbf{1}_{\left\{\tau_{0}^{+}>t\right\}} \mathrm{d} t\right] \\
& +\left(\frac{q \Phi(\theta) \Phi^{\prime}(q+\theta)}{\Phi(q+\theta)(\Phi(q+\theta)-\Phi(\theta))}-\frac{\theta}{\theta+q}\right) \mathrm{E}_{x}\left[\exp \left(-(q+\theta) \tau_{0}^{+}\right)\right] \\
& =1+\frac{\theta}{q+\theta} \mathrm{E}_{x}\left[\exp \left(-(q+\theta) \tau_{0}^{+}\right)\right]-\mathrm{E}_{x}\left[\exp \left(-\theta \tau_{0}^{+}\right)\right] \\
& +\left(\frac{q \Phi(\theta) \Phi^{\prime}(q+\theta)}{\Phi(q+\theta)(\Phi(q+\theta)-\Phi(\theta))}-\frac{\theta}{\theta+q}\right) \mathrm{e}^{\Phi(q+\theta) x} \\
& =1-\mathrm{e}^{\Phi(\theta) x}+\frac{q \Phi(\theta) \Phi^{\prime}(q+\theta)}{\Phi(q+\theta)(\Phi(q+\theta)-\Phi(\theta))} \mathrm{e}^{\Phi(q+\theta) x},
\end{aligned}
$$

which is (13), since $Z_{v}^{(r)}=1$ for all $x \leq 0$ and $v, r \geq 0$.

Proof of (14). We can write the event $\left\{T_{\theta}<e_{q}\right\}$ as a disjoint union:

$$
\left\{\tilde{e}_{\theta}<e_{q}\right\} \cup A^{-} \cup A,
$$

where

$$
A=\left\{\tilde{e}_{\theta}>e_{q}, X_{e_{q}}>0, X_{s} \neq 0 \text { for all } s \in\left[e_{q}, \tilde{e}_{\theta}\right]\right\}
$$

and

$$
A^{-}=\left\{\tilde{e}_{\theta}>e_{q}, X_{s}<0 \text { for all } s \in\left[e_{q}, \tilde{e}_{\theta}\right]\right\} .
$$

Since we already have an expression for $\mathrm{P}_{x}\left(A^{-}\right)=\mathrm{E}_{x}\left[\exp \left(-q \sigma_{\theta}^{+}\right)\right]-\theta /(q+\theta)$, we need only consider $A$. 
First assume that $x \leq 0$. In a similar fashion as in the proof of (20) we deduce from (4), (10), the lack of memory property of the exponential distribution, and the Markov property that

$$
\begin{aligned}
\mathrm{P}_{x}(A) & =q \mathrm{E}_{x}\left[\int_{0}^{\infty} \mathrm{e}^{-q t} \mathbf{1}_{\left\{\tilde{e}_{\theta} \geq t\right\}} \mathbf{1}_{\left\{X_{t}>0, X_{s} \neq 0 \text { for all } s \in\left[t, \tilde{e}_{\theta}\right]\right\}} \mathrm{d} t\right] \\
& =q \int_{0}^{\infty} \mathrm{e}^{-(q+\theta) t} \int_{0}^{\infty} \mathrm{P}_{x}\left(X_{t} \in \mathrm{d} y\right) \mathrm{P}_{y}\left(T(0)>\tilde{e}_{\theta}\right) \mathrm{d} t \\
& =q \int_{0}^{\infty} u^{(q+\theta)}(y-x) \mathrm{P}_{y}\left(T(0)>\tilde{e}_{\theta}\right) \mathrm{d} y \\
& =q \int_{0}^{\infty} \Phi^{\prime}(q+\theta) \mathrm{e}^{-\Phi(q+\theta)(y-x)}\left(1-\mathrm{e}^{\Phi(\theta) y}+\psi^{\prime}(\Phi(\theta)) W^{(\theta)}(y)\right) \mathrm{d} y \\
& =q \Phi^{\prime}(q+\theta) \mathrm{e}^{\Phi(q+\theta) x}\left(\frac{1}{\Phi(q+\theta)}-\frac{1}{\Phi(q+\theta)-\Phi(\theta)}+\frac{1}{q \Phi^{\prime}(\theta)}\right) .
\end{aligned}
$$

We find that

$$
\begin{aligned}
\mathrm{E}_{x}\left[\exp \left(-q T_{\theta}\right)\right]= & \mathrm{P}_{x}(A)+\mathrm{P}_{x}\left(A^{-}\right)+\frac{\theta}{\theta+q} \\
= & q \Phi^{\prime}(q+\theta) \mathrm{e}^{\Phi(q+\theta) x}\left(\frac{1}{\Phi(q+\theta)}-\frac{1}{\Phi(q+\theta)-\Phi(\theta)}+\frac{1}{q \Phi^{\prime}(\theta)}\right) \\
& +1-\mathrm{e}^{\Phi(\theta) x}+\mathrm{e}^{\Phi(q+\theta) x} \frac{q \Phi(\theta) \Phi^{\prime}(q+\theta)}{\Phi(q+\theta)(\Phi(q+\theta)-\Phi(\theta))} \\
= & 1-\mathrm{e}^{\Phi(\theta) x}+\frac{\Phi^{\prime}(q+\theta)}{\Phi^{\prime}(\theta)} \mathrm{e}^{\Phi(q+\theta) x} .
\end{aligned}
$$

Finally, let $x>0$. As before, we find that

$$
\left\{T_{\theta}<e_{q}\right\}=\left\{\tilde{e}_{\theta}<e_{q}\right\} \cup\left\{T(0)>\tilde{e}_{\theta}, \tilde{e}_{\theta} \geq e_{q}\right\} \cup\left(\left\{T(0)<\tilde{e}_{\theta}\right\} \cap\left(A \cup A^{-}\right)\right) .
$$

An application of the strong Markov property at $T(0)$, the memoryless property of the exponential distribution, (7), (8), and (10) imply that

$$
\begin{aligned}
\mathrm{E}_{x}\left[\exp \left(-q T_{\theta}\right)\right]= & \frac{\theta}{\theta+q}+\mathrm{E}_{x}\left[\left(\exp \left(-\theta e_{q}\right)-\mathrm{e}^{-\theta T(0)}\right) \mathbf{1}_{\left\{T(0)>e_{q}\right\}}\right] \\
& +\mathrm{P}_{x}\left(T(0)<e_{q} \wedge \tilde{e}_{\theta}\right) \mathrm{P}\left(T_{\theta}<e_{q}<\tilde{e}_{\theta}\right) \\
= & 1+\frac{\theta}{\theta+q} \mathrm{E}_{x}\left[\mathrm{e}^{-(q+\theta) T(0)}\right]-\mathrm{E}_{x}\left[\mathrm{e}^{-\theta T(0)}\right] \\
& +\mathrm{E}_{x}\left[\mathrm{e}^{-(q+\theta) T(0)}\right]\left(\frac{\Phi^{\prime}(q+\theta)}{\Phi^{\prime}(\theta)}-\frac{\theta}{\theta+q}\right) \\
= & 1-\mathrm{e}^{\Phi(\theta) x}+\frac{1}{\Phi^{\prime}(\theta)}\left(W^{(\theta)}(x)-W^{(q+\theta)}(x)\right)+\frac{\Phi^{\prime}(q+\theta)}{\Phi^{\prime}(\theta)} \mathrm{e}^{\Phi(q+\theta) x},
\end{aligned}
$$

which completes the proof of Theorem 2.

Remark 3. Two of the main ingredients in the proof of Theorem 2 are the $q$-potential measure of $X$ and the Laplace transform of the first passage time above or below a given level. These quantities are also known for certain Lévy processes which do have positive jumps. 
Proposition 2 of [1] indicates that results similar to (11) and (13) can be obtained for so-called phase-type Lévy processes. Similarly, as mentioned before Corollary 2, we can use the scaling property to find the Laplace exponent of the last hitting time of 0 for any stable process with index $\alpha>1$. See the proof of Lemma VIII.13 of [2] for details.

Remark 4. As mentioned in the introduction, result (11) could be useful in risk theory, since it gives information about the last time when the risk process is negative before an independent, exponentially distributed time. Indeed, the last passage of $X$ below 0 before a fixed time horizon can be found by inverting the double Laplace transform in (11). Unfortunately, this seems to be tractable analytically only in very specific cases. An additional complication is that the scale function is not always available explicitly. We refer the reader to [9] for examples of explicit examples of scale functions. Furthermore, scale functions can be evaluated numerically and we refer the reader to [17] and [18] for such numerical schemes.

\section{Application: an extension of a result of Doney [6]}

Doney [6, Corollary 3] showed that, for a spectrally negative stable process with index $\alpha$, it holds that

$$
\mathrm{P}\left(X_{t}=\bar{X}_{t}=t \text { for some } 0<t<\infty\right)=\frac{1}{\alpha} .
$$

In this section we extend this result and, in particular, we find (for a general spectrally negative Lévy process) the Laplace exponent of the random time $\tau_{1}$ defined by

$$
\tau_{1}:=\sup \left\{t \geq 0: X_{t}=\bar{X}_{t}=t\right\}
$$

recalling the convention that $\sup \varnothing=0$. Similarly, we define

$$
\begin{aligned}
& \tau_{2}=\sup \left\{t \geq 0: X_{t}=t\right\}, \\
& \tau_{3}=\sup \left\{t \geq 0: X_{t} \geq t\right\}, \\
& \tau_{4}=\sup \left\{t \geq 0: \bar{X}_{t} \geq t\right\} .
\end{aligned}
$$

Since

$$
\left\{t \geq 0: X_{t}=\bar{X}_{t}=t\right\} \subseteq\left\{t \geq 0: X_{t}=t\right\} \subseteq\left\{t \geq 0: X_{t} \geq t\right\} \subseteq\left\{t \geq 0: \bar{X}_{t} \geq t\right\},
$$

we have

$$
\tau_{1} \leq \tau_{2} \leq \tau_{3} \leq \tau_{4} .
$$

These random times are trivial when $X$ is of bounded variation with drift $d \leq 1$ (since they are all equal to the first jump time when $d=1$ and all equal to 0 when $d<1$ ) and, hence, we assume throughout this section that

$$
d>1 \text { whenever } X \text { is of bounded variation. }
$$

Let $q>0$. Since

$$
\lim _{\lambda \rightarrow \infty} \frac{\psi(\lambda)}{\lambda}= \begin{cases}\infty & \text { when } X \text { is of unbounded variation, } \\ d & \text { when } X \text { is of bounded variation with drift } d,\end{cases}
$$

we see that there exists a unique $y_{q}>0$ such that

$$
\psi\left(y_{q}\right)=q+y_{q} .
$$


Now let $z_{q}:=\psi\left(y_{q}\right)$. Then $\Phi\left(z_{q}\right)=\Phi\left(\psi\left(y_{q}\right)\right)=y_{q}=\psi\left(y_{q}\right)-q=z_{q}-q$. Finally, set

$$
y_{0}:= \begin{cases}0 & \text { when } \psi^{\prime}(0) \geq 1 \\ y & \text { when } \psi^{\prime}(0)<1\end{cases}
$$

where $y$ is the unique solution on $(0, \infty)$ of $\psi(\lambda)=\lambda$ when $\psi^{\prime}(0)<1$. We can use Theorem 2 to establish the following result.

Corollary 3. Suppose that $X$ is a spectrally negative Lévy process which is of unbounded variation or of bounded variation with drift $d>1$. Then

$$
\begin{aligned}
\mathrm{E}\left[\exp \left(-q \tau_{1}\right)\right] & =\frac{\psi^{\prime}\left(y_{q}\right)}{\psi^{\prime}\left(y_{0}\right)} \frac{\psi^{\prime}\left(y_{0}\right)-1}{\psi^{\prime}\left(y_{q}\right)-1}, \\
\mathrm{E}\left[\exp \left(-q \tau_{2}\right)\right] & =\frac{\psi^{\prime}\left(y_{0}\right)-1}{\psi^{\prime}\left(y_{q}\right)-1}, \\
\text { and } \mathrm{E}\left[\exp \left(-q \tau_{3}\right)\right] & =\frac{q y_{0}}{y_{q}\left(y_{q}-y_{0}\right)\left(\psi^{\prime}\left(y_{q}\right)-1\right)} .
\end{aligned}
$$

Finally,

$$
\mathrm{E}\left[\exp \left(-q \tau_{4}\right)\right]=\frac{q y_{0} \psi^{\prime}\left(y_{q}\right)}{\psi\left(y_{q}\right)\left(\psi\left(y_{q}\right)-y_{0}\right)\left(\psi^{\prime}\left(y_{q}\right)-1\right)} .
$$

Proof. First, suppose that $X$ does not drift to $-\infty$. Introduce the processes $Y_{t}=X_{t}-t$ and $Z_{t}=t-\tau_{t}^{+}$, which are both spectrally negative Lévy processes. The assumption that $X$ does not drift to $-\infty$ is used here to ensure that $\mathrm{P}\left(\tau_{t}^{+}<\infty\right)=1$. Note that, since

$$
\left\{t \geq 0: X_{t}=\bar{X}_{t}=t\right\}=\left\{t \geq 0: \tau_{t}^{+}=t\right\}
$$

the random times $\tau_{1}$ and $\tau_{2}$ are the last hitting times $T$ of 0 for $Z$ and $Y$, respectively, and $\tau_{3}$ and $\tau_{4}$ are the last passage times above 0 of $Y$ and $Z$, respectively. Using obvious notation, it holds that $\psi^{Y}(\lambda)=\psi(\lambda)-\lambda$ and $\psi^{Z}(\lambda)=\lambda-\Phi(\lambda)$; hence,

$$
\Phi^{Y}(q)=y_{q} \quad \text { and } \quad \Phi^{Z}(q)=z_{q} .
$$

From the implicit function theorem we find that

$$
\frac{\mathrm{d}}{\mathrm{d} q} y_{q}=\frac{1}{\psi^{\prime}\left(y_{q}\right)-1}
$$

and that

$$
\frac{\mathrm{d}}{\mathrm{d} q} z_{q}=\frac{1}{1-\Phi^{\prime}\left(z_{q}\right)}=\frac{\psi^{\prime}\left(y_{q}\right)}{\psi^{\prime}\left(y_{q}\right)-1} .
$$

The result now follows by taking $\theta=0$ and $x=0$ in Theorem 2 .

When $X$ does drift to $-\infty,(28)$ and (29) still hold, but in this case $\tau_{t}^{+}$is a subordinator killed at exponential rate $\Phi(0)$, which is strictly positive as $\psi^{\prime}(0)<0$. Hence, we are now looking for the last passage times before $e_{\Phi(0)}$ of a Lévy process with Laplace exponent given by $\lambda-\Phi(\lambda)+\Phi(0)$. Statements (27) and (30) now follow by an application of Theorem 2 with $\theta=\Phi(0)$ and $x=0$. 
Define, for $s \geq 0$,

$$
A_{s}:=\left\{\text { there exists some } t>s: X_{t}=\bar{X}_{t}=t\right\},
$$

and denote $A=A_{0}$. Equation (25) is contained in the following corollary.

Corollary 4. For a spectrally negative Lévy process, it holds that

$$
\mathrm{P}(A)= \begin{cases}\frac{1}{\psi^{\prime}\left(y_{0}\right)} & \text { when } X \text { has unbounded variation, } \\ \frac{d-\psi^{\prime}\left(y_{0}\right)}{d \psi^{\prime}\left(y_{0}\right)-\psi^{\prime}\left(y_{0}\right)} & \text { when } X \text { has bounded variation with drift } d>1,\end{cases}
$$

where $y_{0}$ is defined in (26). In particular, $\mathrm{P}(A)=1 / \alpha$ for a spectrally negative stable process of index $\alpha$. Also,

$$
\mathrm{P}(A)=1 \quad \Longleftrightarrow \quad \psi^{\prime}(0)=1
$$

In fact, when $\psi^{\prime}(0)=1$,

$$
\mathrm{P}\left(A_{s}\right)=1 \text { for all } s \geq 0 \text {. }
$$

Proof. Since

$$
\lim _{\lambda \rightarrow \infty} \psi^{\prime}(\lambda)= \begin{cases}\infty & \text { when } X \text { is of unbounded variation, } \\ d & \text { when } X \text { is of bounded variation with drift } d,\end{cases}
$$

it follows from Corollary 3 that

$$
\begin{aligned}
\mathrm{P}(A) & =1-\mathrm{P}\left(\tau_{1}=0\right) \\
& =1-\lim _{q \rightarrow \infty} \mathrm{E}\left[\exp \left(-q \tau_{1}\right)\right] \\
& =1-\lim _{q \rightarrow \infty} \frac{\psi^{\prime}\left(y_{q}\right)}{\psi^{\prime}\left(y_{0}\right)} \frac{\psi^{\prime}\left(y_{0}\right)-1}{\psi^{\prime}\left(y_{q}\right)-1} \\
& = \begin{cases}\frac{1}{\psi^{\prime}\left(y_{0}\right)} & \text { when } X \text { is of unbounded variation, } \\
\frac{d-\psi^{\prime}\left(y_{0}\right)}{d \psi^{\prime}\left(y_{0}\right)-\psi^{\prime}\left(y_{0}\right)} & \text { when } X \text { is of bounded variation with drift } d>1 .\end{cases}
\end{aligned}
$$

When $X$ is a stable process of index $\alpha \in(1,2]$, we have $y_{0}=1$ and, thus,

$$
\mathrm{P}(A)=\frac{1}{\psi^{\prime}(1)}=\frac{1}{\alpha} .
$$

To show (31), suppose that $\psi^{\prime}(0)=1$. It then holds that $y_{0}=1$ and, hence, $\mathrm{P}(A)=1$.

For the other direction, we remark that $\psi^{\prime}(0)>1$ implies that $\psi^{\prime}\left(y_{0}\right)=\psi^{\prime}(0)>1$. Also, when $\psi^{\prime}(0)<1$, we have $\psi^{\prime}\left(y_{0}\right)>1$, as $y_{0}$ is the unique solution to $\psi(y)=y$ on $(0, \infty)$ and because $\psi$ is a strictly convex function on $[0, \infty)$. We conclude that, whenever $\psi^{\prime}(0) \neq 1$, we have $\psi^{\prime}\left(y_{0}\right)>1$, from which it follows that $\mathrm{P}(A)<1$.

From (27) we see that $\psi^{\prime}(0)=1$ implies that $\mathrm{E}\left[\exp \left(-q \tau_{1}\right)\right]=0$ for any $q>0$. The final statement in Corollary 4 now follows. 
Remark 5. As an example, we consider a standard Brownian motion. Owing to its continuous paths we have $\tau_{2}=\tau_{3}$. Its Laplace exponent is given by $\psi(\lambda)=\lambda^{2} / 2$, so $\psi_{Y}(\lambda)=\lambda^{2} / 2-\lambda$ and $\psi_{Z}(\lambda)=\lambda-\sqrt{2 \lambda}$; hence,

$$
y_{q}=1+\sqrt{2 q+1} \text { and } z_{q}=1+q+\sqrt{2 q+1} .
$$

From Corollary 3 we readily deduce that

$$
\begin{aligned}
\mathrm{E}\left[\exp \left(-q \tau_{1}\right)\right] & =\frac{a_{q}+1}{2 a_{q}}, \\
\mathrm{E}\left[\exp \left(-q \tau_{2}\right)\right] & =\mathrm{E}\left[\exp \left(-q \tau_{3}\right)\right]=\frac{1}{a_{q}}, \\
\text { and } \quad \mathrm{E}\left[\exp \left(-q \tau_{4}\right)\right] & =\frac{2 a_{q}+2}{(q+2) a_{q}+4 q+2},
\end{aligned}
$$

where $a_{q}=\sqrt{2 q+1}$.

\section{Appendix A}

Proof of Lemma 1. Suppose that $q>0$. First, let $\lambda>0$. Then (6) follows by integration by parts. Indeed, in this case

$$
\begin{aligned}
\int_{[0, \infty)} \mathrm{e}^{-\lambda x} W_{\Phi(q)}(\mathrm{d} x) & =\lambda \int_{0}^{\infty} \mathrm{e}^{-\lambda x} W_{\Phi(q)}(x) \mathrm{d} x \\
& =\frac{\lambda}{\psi_{\Phi(q)}(\lambda)} \\
& =\frac{\lambda}{\psi(\Phi(q)+\lambda)-q} .
\end{aligned}
$$

Under $\mathrm{P}_{\Phi(q)}$, the process $\left\{X_{t}\right\}_{t \geq 0}$ drifts to $\infty$ and now from Equation (8.15) of [13] we deduce that

$$
W_{\Phi(q)}(x)=\frac{1}{\psi_{\Phi(q)}^{\prime}(0+)} \mathrm{P}_{x}\left(\inf _{t \geq 0} X_{t} \geq 0\right) .
$$

It follows that

$$
\lim _{x \rightarrow \infty} W_{\Phi(q)}(x)=\Phi^{\prime}(q),
$$

and, hence, (6) holds for $\lambda=0$ as well.

Next, we show that (6) holds for $\lambda=-\Phi(q)$. We make use of the resolvent measure for the reflected process $\left\{V_{t}\right\}_{t \geq 0}$ defined by

$$
V_{t}=\sup _{0 \leq s \leq t}\left(X_{s} \vee 0\right)-X_{t} .
$$

In Theorem 1(ii) of [16], the resolvent measure

$$
R_{a}^{q}(x, \mathrm{~d} y)=\int_{0}^{\infty} \mathrm{e}^{-q t} \mathrm{P}_{x}\left(V_{t} \in \mathrm{d} y, \sup _{0 \leq s \leq t} V_{s} \leq a\right)
$$

of $V$ killed at exceeding a certain level $a>0$ was found. In particular, for $x=0$, it holds that

$$
R_{a}^{q}(0, \mathrm{~d} y)=\left(W^{(q)}(a) \frac{W^{(q)^{\prime}}(y+)}{W^{(q)^{\prime}}(a+)}-W^{(q)}(y)\right) \mathrm{d} y \quad \text { for } y \in(0, a]
$$


and $R_{a}^{q}(0,\{0\})=W^{(q)}(a) W^{(q)}(0) / W^{(q) \prime}(a+)$. Using the fact that $W_{\Phi(q)}(\infty)<\infty$ and (5), we can take $a \rightarrow \infty$ and deduce that the resolvent measure

$$
R^{q}(0, \mathrm{~d} y)=\int_{0}^{\infty} \mathrm{e}^{-q t} \mathrm{P}_{0}\left(V_{t} \in \mathrm{d} y\right)
$$

of the unkilled reflected process is given by

$$
R^{q}(0, \mathrm{~d} y)=\left(\frac{1}{\Phi(q)} W^{(q)^{\prime}}(y+)-W^{(q)}(y)\right) \mathrm{d} y=\frac{1}{\Phi(q)} \mathrm{e}^{\Phi(q) y} W_{\Phi(q)}(\mathrm{d} y) \quad \text { for } y \geq 0 .
$$

An application of Fubini's theorem yields

$$
\begin{aligned}
\int_{[0, \infty)} \mathrm{e}^{\Phi(q) x} W_{\Phi(q)}(\mathrm{d} x) & =\Phi(q) \int_{[0, \infty)} R^{q}(0, \mathrm{~d} x) \\
& =\Phi(q) \int_{[0, \infty)} \int_{0}^{\infty} \mathrm{e}^{-q t} \mathrm{P}\left(V_{t} \in \mathrm{d} x\right) \mathrm{d} t \\
& =\Phi(q) \int_{0}^{\infty} \mathrm{e}^{-q t} \mathrm{P}\left(V_{t} \in[0, \infty)\right) \mathrm{d} t \\
& =\frac{\Phi(q)}{q}
\end{aligned}
$$

which is (6) for $\lambda=-\Phi(q)$.

Finally, for the case $-\Phi(q)<\lambda<0$, we make use of an analytic extension. We can extend the Laplace exponent $\psi$ to those $z \in \mathbb{C}$ for which $\operatorname{Re}(z)>0$ and we denote this extension by $\Psi$. Define the function $g: A \rightarrow \mathbb{C}$ by

$$
g(z)= \begin{cases}\frac{z}{\Psi(z+\Phi(q))-q} & \text { when } z \neq 0 \text { and } \operatorname{Re}(z)>-\Phi(q), \\ \Phi^{\prime}(q) & \text { when } z=0\end{cases}
$$

where $A$ is an open set in $\mathbb{C}$ such that $\operatorname{Re}(z)>-\Phi(q)$ for all $z \in A$ and such that $\Psi(z+\Phi(q)) \neq$ $q$ on $A \backslash\{0\}$. Since the Laplace exponent $\Psi$ is analytic when $\operatorname{Re}(z)>0$, we can write

$$
\Psi(z+\Phi(q))=q+\sum_{k=1}^{\infty} \frac{z^{k}}{k !} \Psi^{(k)}(\Phi(q)) \quad \text { when } \operatorname{Re}(z)>-\Phi(q),
$$

where $\Psi^{(k)}$ denotes the $k$ th derivative of $\Psi$. The fact that $\psi^{\prime}(\Phi(q))>0$ implies that $g$ is bounded in some (complex) neighborhood of 0 , and we can use the Riemann removable singularity theorem to deduce that $g(\lambda)$ is real analytic for $\lambda>-\Phi(q)$. The coefficients $c_{n}$ in the power series of $g$ are given in terms of the $n$th (right) derivative at 0 of the left-hand side of (32). Specifically, because of (34),

$$
c_{n}=\int_{[0, \infty)} \frac{(-x)^{n}}{n !} W_{\Phi(q)}(\mathrm{d} x) \quad \text { for } n \in \mathbb{N} .
$$

In particular, for $\lambda \in(-\Phi(q), 0)$,

$$
g(\lambda)=\sum_{n=0}^{\infty} \lambda^{n} \int_{[0, \infty)} \frac{(-x)^{n}}{n !} W_{\Phi(q)}(\mathrm{d} x)=\sum_{n=0}^{\infty} \int_{[0, \infty)} \frac{|\lambda x|^{n}}{n !} W_{\Phi(q)}(\mathrm{d} x) .
$$


From another application of Fubini's theorem, it follows that, for any $|\lambda|<\Phi(q)$,

$$
\begin{aligned}
g(\lambda) & =\sum_{n=0}^{\infty} c_{n} \lambda^{n} \\
& =\sum_{n=0}^{\infty} \lambda^{n} \int_{[0, \infty)} \frac{(-x)^{n}}{n !} W_{\Phi(q)}(\mathrm{d} x) \\
& =\int_{[0, \infty)} \sum_{n=0}^{\infty} \frac{(-\lambda x)^{n}}{n !} W_{\Phi(q)}(\mathrm{d} x) \\
& =\int_{[0, \infty)} \mathrm{e}^{-\lambda x} W_{\Phi(q)}(\mathrm{d} x) .
\end{aligned}
$$

This completes the proof of Lemma 1.

\section{Acknowledgements}

This paper was partly written during my visit to the University of Bath and I would like to express my gratitude for their hospitality and support. I would also like to thank an anonymous referee for carefully reading an earlier version of this paper and for useful comments.

\section{References}

[1] Asmussen, S., Avram, F. And Pistorius, M. R. (2004). Russian and American put options under exponential phase-type Lévy models. Stoch. Process. Appl. 109, 79-111.

[2] Bertoin, J. (1996). Lévy Processes (Camb. Tracts Math. 121). Cambridge University Press.

[3] Bingham, N. H. (1975). Fluctuation theory in continuous time. Adv. Appl. Prob. 7, 705-766.

[4] Chan, T. (2004). Some applications of Lévy processes in insurance and finance. Finance 25, 71-94.

[5] Chiu, S. N. AND Yin, C. (2005). Passage times for a spectrally negative Lévy process with applications to risk theory. Bernoulli 11, 511-522.

[6] Doney, R. A. (1991). Hitting probabilities for spectrally positive Lévy processes. J. London Math. Soc. 44, 566-576.

[7] Emery, D. J. (1973). Exit problems for a spectrally positive process. Adv. Appl. Prob. 5, 498-520.

[8] Gerber, H. U. (1990). When does the surplus reach a given target? Insurance Math. Econom. 9, 115-119.

[9] Hubalek, F. and Kyprianou, A. E. (2007). Old and new examples of scale functions for spectrally negative Lévy processes. Preprint. Available at http://arxiv.org/abs/0801.0393.

[10] HuZaK, M., Perman, M., ŠIKIĆ, H. AND VondračEK, Z. (2004). Ruin probabilities and decompositions for general perturbed risk processes. Ann. Appl. Prob. 14, 1378-1397.

[11] Huzak, M., Perman, M., ŠIKić, H. and VondračEK, Z. (2004). Ruin probabilities for competing claim processes. J. Appl. Prob. 41, 679-690.

[12] Klüppelberg, C., Kyprianou, A. E. And Maller, R. A. (2004). Ruin probabilities and overshoots for general Lévy insurance risk processes. Ann. Appl. Prob. 14, 1766-1801.

[13] Kyprianou, A. E. (2006). Introductory Lectures on Fluctuations of Lévy Processes with Applications. Springer, Berlin.

[14] LÉvy, P. (1965). Processus Stochastiques et Mouvement Brownien, 2nd edn. Gauthier-Villars, Paris.

[15] Lundberg, F. (1903). Approximerad Framställning av Sannolikehets-Funktionen. Återförsäkering av Kollektivrisker. Almqvist \& Wiksell, Uppsala.

[16] Pistorius, M. R. (2004). On exit and ergodicity of the spectrally one-sided Lévy process reflected at its infimum. J. Theoret. Prob. 17, 183-220.

[17] Rogers, L. C. G. (2000). Evaluating first-passage probabilities for spectrally one-sided Lévy processes. J. Appl. Prob. 37, 1173-1180.

[18] Surya, B. A. (2008). Evaluating scale functions of spectrally negative Lévy processes. J. Appl. Prob. 45, 135149. 